\title{
De la nouvelle à l'essai, au seizième siècle*
}

\author{
ANDRÉ BERTHIAUME
}

$\mathrm{C}$

et article voudrait alimenter tant soit peu la réflexion sur l'évolution des formes littéraires au seizième siècle.

Convenons d'abord qu'il est à la fois plus facile et plus difficile d'étudier un texte ancien qu'un texte contemporain. Nous avons le recul nécessaire, mais au prix d'une disjonction d'avec le contexte socio-culturel de l'époque. C'est sans doute en dépit de cette tension que nous proposons nos lectures ... et que je risquerai ici une comparaison entre deux oeuvres marquantes de la Renaissance française: l'Heptaméron de Marguerite de Navarre (1559) et les Essais de Montaigne (1580-1595). Je devine sans peine ce qu'il peut y avoir de périlleux à rapprocher deux oeuvres si différentes à maints égards,-mais le sont-elles à ce point puisqu'une vintaine d'années seulement séparent leur publication? J'ajouterai que des critiques ont déjà établi ce lien mais dans d'autres perspectives que la mienne.

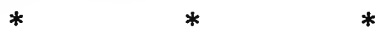

Les premières manifestations de la nouvelle française nous conduisent inévitablement à l'Heptaméron, ${ }^{1}$ texte inachevé qui parut dix ans après la mort de Marguerite, avec un titre qui n'était pas d'elle mais de l'éditeur Claude Gruget, recueil où par ailleurs on ne semble pas faire de différence entre nouvelles, histoires et "comptes" (p.720). Comiques ou tragiques, les histoires-dont certaines préfigurent les Chroniques italiennes de Stendhaldéveloppent les thèmes traditionnels de la "vertu d'amour qui se faict sentir quand elle n'est point faincte" (p.724) et des tribulations prétendument scandaleuses de l'état monastique. ${ }^{2}$ La structure du recueil retient l'attention, on le sait, autant que son contenu. Structure, disposition redevable au Décaméron, que Parlamente appelle "les cent Nouvelles de Bocace" (p. 709). Son encadrement est remarquable par ce prologue qui accumule les anecdotes dont, incidemment, la violence étonne le lecteur d'aujourd'hui: l'utilisation outrancière des armes stupéfie davantage que les dégâts somme toute mineurs d'une inondation: c'est l'épée qui est à l'origine d'une véritable hécatombe, 
pas l'eau. ${ }^{3}$ Mais l'examen de la dialectique des armes et des lettres nous éloignerait de notre sujet.

Revenons à un autre aspect de l'organisation interne qui caractérise l'Heptaméron: les narrations d'histoires à pleurer ou à rire, dites et redites "véritables," sont suivies de commentaires, plus spécifiquement d'échanges entre dix "devisants"—ou "opinants" (p. 708), terme utilisé par Oisille. Dialogues souvent animés, vifs, voix souvent discordantes, contradictoires. Jean Rousset voit dans ces débats de "véritables protocoles de lecture, où les acteurs se font glossateurs de leurs récits." "Ainsi, l'Heptaméron nous convie à la rencontre de deux genres, sinon de deux styles..$^{5} \mathrm{~d}$ 'une part, le récit court, émanation du fabliau, de l'exemplum médiéval et de la novella italienne; ${ }^{6}$ d'autre part, l'entretien, dont les antécédents sont immémoriaux, platoniciens, que l'on peut rapprocher du célèbre Cortegiano, plusieurs fois traduit en français à partir de 1537 .

On retiendra aussi dans l'Heptaméron une certaine visibilité des "coutures," pour utiliser un terme cher à Montaigne, c'est-à-dires une certaine lisibilité des liaisons, de ces incessants passages du narratif au réflexif. En effet, les codes narratif et argumentatif, tout en commandant des écritures différentes, sont passablement étanches; les changements de narrateur sont d'ailleurs ostentatoires: après chaque récit sagement linéaire, on donne explicitement sa voix à l'autre:

"Pour n'en debatre plus, je vous prie, Parlamente, donnez vostre voix à quelcun.-Je la donne très volontiers, ce dist-elle, à Symontault; car après ces deux tristes nouvelles, il ne fauldra de nous en dire une qui ne nous fera poinct pleurer.-Je vous remercie, dist Simontault; en me donnant vostre voix, il ne s'en fault gueres que ne me nommez plaisant, qui est ung nom que je trouve fort fascheux ..." (p. 805)

Le don de sa voix ou la réception de ce legs est un leitmotiv qui court tout le long du recueil. Donc importance des fonctions extra-narratives que Gérard Genette a appelées d'attestation ou de régie. ${ }^{7}$ En revanche, on a déjà noté que si les devisants sont bien présents dans leurs commentaires, ils sont plutôt discrets quand ils adoptent le statut de narrateurs, ${ }^{8}$ sans doute pour laisser plus de latitude, donner plus de relief aux interprétations qui suivent.

Étanchéité relative des codes, fonctions énonciatrices bien délimitées, passage de l'objectivité à la subjectivité, on reconnaîtra que, dans une perspective homologique, ces caractéristiques conviennent fort bien à un univers cloisonné, hiérarchisé, tant socialement que spirituellement, voué à l'édification, à l'élévation, tendu vers le haut, sinon le Très-Haut: la femme 
du muletier qui va mourir pour sauver son honneur a "les oeilz eslevez au ciel" (p. 720). Une critique en arrive même à la conclusion que "l'action de l'Heptaméron [n'est rien d'autre que] l'histoire de la conversion, par Oisille, des autres devisants." 9

Ce qui frappe donc, c'est l'importance, quantitative autant que qualitative, du réflexif, du doxologique ${ }^{10}$ dans l'économie du recueil et, par voie de conséquence, son caractère didactique: "Voylà, mes dames, proclame le timide Saffredent, une histoire que voluntiers je vous monstre icy pour exemple à fin que, quand vos mariz vous donnent des cornes de cheuvreux, vous leur en donnez de cerf." (p. 726) ${ }^{11}$ Il y a en effet accumulation d'exempla mais, tout compte fait, absence de synthèse, ouverture puisque le "passetemps" (p. 707), leitmotiv du prologue, doit persister; un passe-temps utile, instructif, voire édifiant, sorte de succédané des lectures commentées de la Bible, "leçons" justement louangées dans le prologue (p. 707). ${ }^{12}$

Dans la première oeuvre intitulée Essais, ${ }^{13}$ où un "je" s'exerce en marge de ses lectures, s'expérimente à loisir, le discours argumentatif, enthymématique selon Roland Barthes, ${ }^{14}$ occupe évidemment une place prépondérante, d'ailleurs annoncé par le titre. Mais il faut noter que le narratif n'est pas évacué, il s'en faut. Antoine Compagnon voit même “deux écritures dans les Essais, une écriture courte, pointue, elliptique, et une écriture longue, balancée et prolixe, pour les anecdotes, intimes et historiques." 15 Même style partagé que dans l'Heptaméron. Soulignons l'importance de l'anecdote, du petit fait curieux, de l'historiette pour stimuler la pensée, la rendre plus concrète, relancer l'essai montaignien qui, en 1580, était remarquable par sa brièveté. ${ }^{16}$ Montaigne dira dans un ajout de 1588 'qu'il n'est rien si contraire à [son] stile qu'une narration estendue" (I, 21, p. 105). Il existe d'ailleurs plusieurs études sur le Montaigne conteur. ${ }^{17}$ Hugo Friedrich voyait même en l'essayiste "un maître du récit anecdotique."18

La thématique n'est évidemment pas la même chez la soeur de François $1^{\text {er }}$ et chez le gentilhomme de Bordeaux, mais il y a des traits communs: les deux auteurs montrent leur intérêt pour les jeux aristocratiques de l'être et du paraître, pour les motivations psychologiques: les ressorts de la passion amoureuse sont scrutés chez Marguerite, ceux de l'ambition ou de la vanité chez Montaigne. Et l'on peut imaginer sans peine que l'auteure de l'Heptaméron aurait apprécié et mis à profit maintes anecdotes rapportées dans les Essais. Tout en préservant leur attachement aux valeurs traditionnelles, religieuses et politiques, les deux écrivains accordent la plus 


\section{8 / Renaissance and Reformation}

grande importance à "la pluralité d'opinions," expression utilisée par Oisille (p. 708). La notion d'expérience est mise de l'avant dès le départ dans l'Heptaméron (Oisille est présentée comme une "dame vefve, de longue experience," p. 702); elle donne son titre au dernier essai de Montaigne. ... Curieusement les deux auteurs nobles se signalent par leur méfiance à l'égard de "la beaulté de la rhetoricque" (p. 709), d'où son corollaire: l'adoption d'un style bas, humble, "simple et naïf" (I, 26, p.171), proche de l'oral, de la conversation, de cet "art de conférer" célébré par Montaigne.

Par ailleurs, l'essayiste, qui qualife l'Heptaméron de "gentil livre pour son étoffe" (II, p. 108), c'est-à-dire de noble livre pour sa matière, pour son contenu, se préoccupe moins de l'authenticité de ses sources ${ }^{19}$ que la reine de Navarre: "dira chascun, décrète Parlamente, quelque histoire qu'il aura veue ou bien oy dire à quelque personne digne de foy." (p. 709) Encore que Marguerite, selon Nicole Cazauran, ne fit là qu'obéir à une convention. ${ }^{20}$

Il importe donc surtout de noter la proximité, la cohabitation du narratif et du réflexif, du raconter et du persuader dans deux oeuvres importantes de la seconde moitié du seizième siècle, avec des tonalités et des perspectives propres à chacune. Le narratif et l'argumentatif sont ici dynamiquement liés, en interaction dans une prose discontinue. Henri Coulet décrit ainsi la dialectique propre à l'Heptaméron: “ ... l'expérience, c'est-à-dire le récit, suscite le heurt des convictions; le heurt des convictions renvoie à une nouvelle expérience." 21 On conviendra que cette description s'applique aussi fort bien à la dynamique inhérente aux Essais. En somme, ceux-ci ne sont-ils pas déjà formellement présents, inscrits dans l'Heptaméron? L'attitude désinvolte de Montaigne à l'égard de son lecteur dont "dès l'entrée" (p. 9) il prétend prendre congé ne doit pas nous donner le change, quant au caractère apparemment non didactique de son oeuvre. Cet avis liminaire est démenti tout le long des Essais par l'utilisation constante des pronoms vous et nous. Comme si l'argumentatif pouvait se passer d'un destinataire!

Ainsi, de la nouvelle à l'essai, d'une forme parcellaire à une autre, on retrouve la même double composante fondamentale, la même dialectique de la narration et du commentaire, la même matrice rhétorique, avec un je apparemment singulier chez Montaigne,-—n réalité tout aussi pluriel, polyphonique que dans l'Heptaméron, où, par ailleurs, les voix, "multiples et incertaines,"22 importent plus que les personnages, toujours selon Nicole Cazauran. ${ }^{23}$

Il y a donc, me semble-t-il, continuité formelle entre l'Heptaméron et les Essais, même si les contextes socio-culturels different dans une certaine mesure. Pour sa part, Montaigne met l'accent sur l'argumentatif, revendique 
davantage "les discours" que "les exemples," préfère raconter "les choses passées que présentes" (I,21, p. 104); enfin, il prend plaisir à gommer les "coutures," ce qui amène Jean Terrasse, dans son excellent chapitre sur "Des coches," à conclure de la façon suivante: "Comme les transitions sont aussi importantes que les éléments qu'elles relient, le lecteur ne distingue plus les idées principales des idées secondaires, car il n'y a plus entre elles de hiérarchie. [...] Toutes les faces de l'objet sont étalées et mises sur le même plan." 24 C'est comme si, de l'Heptaméron aux Essais, l'on passait d'une écriture verticale à une écriture horizontale. Tout compte fait, les lettres a,b,c des éditions modernes des Essais nous leurrent car, avec ses "alongeails" et ses citations éclairs, le discours montaignien abolit non seulement toute hiérarchie de plans mais aussi toute temporalité.

Montaigne et Marguerite de Navarre sont à leur façon des compilateurs, des praticiens d'un discours qui se nourrit d'additions, de juxtapositions; des glossateurs qui se situent dans cette tradition humaniste du discours discontinu, "très longtemps considéré, comme le note Jean Lafond, que comme l'échec du continu." 25 Derrière la quête de l'anecdote qui nourrit l'oeuvre fragmentaire, une pensée se cherche, une écriture se trouve. On sait que la curiositas et le doute traversent toute la Renaissance, impliquant une remise en cause d'"une certaine idée de la totalisation du sens. ${ }^{26}$ "Friedrich relie le fragmentarisme au scepticisme. ${ }^{27}$ L'oeuvre narrative de Marguerite de Navarre reste-involontairement, il est vrai-inachevée: 72 nouvelles sur les 100 prévues; par ailleurs, chez Montaigne comme chez d'autres auteurs de la Renaissance, l'inachèvement est une forme d'achèvement. Marcel Tetel situe volontiers l'auteure de l'Heptaméron entre Rabelais-en particulier celui du Tiers Livre, dédié précisément à Marguerite-et Montaigne, ${ }^{28}$ à cause de leur propension à l'ambiguïté. Mais ce n'est pas seulement une affaire de contenu. L'Heptaméron, qualifié par Montaigne de "gentil livre" pour sa matière, est également intéressant pour sa manière: cette oeuvre ne contient-elle pas les principaux traits formels de l'essai montaignien? Elle n'est évidemment pas la seule à s'inscrire dans ce courant où le narratif va de pair avec l'argumentatif-que l'on pense seulement aux Dialogues non moins profitables que facétieux (1565) de Jacques Tahureau-mais il m'a paru important, ne serait-ce qu'à cause de ses implications pédagogiques, de revenir brièvement sur cette filiation particulière.

Université Laval 


\section{0 / Renaissance and Reformation}

\section{Notes}

* Cet article a d'abord été lu dans le cadre de la rencontre annuelle de la Société canadienne d'études de la Renaissance, le 31 mai 1989, à l'Université Laval.

1. Marguerite de Navarre, L'Heptaméron, dans Conteurs français du XVIe siècle, textes présentés et annotés par Pierre Jourda. Paris, Gallimard, Bibliothèque de la Pléiade, 1965. Mes références renvoient à cette édition.

2. Voir Isida Cremona, "Les cordeliers et la notion de justice dans l'Heptaméron de Marguerite de Navarre, dans La Nouvelle, Montréal, Plato Academic Press, 1983. p. 218.

3. Sur le thème de la violence, voir Nicole Cazauran, L'Heptaméron de Marguerite de Navarre, Paris, SEDES (Société d'Édition d'Enseignement Supérieur), 1976, p. 60 , 135.

4. Jean Rousset, "La question du narrataire," dans Problèmes actuels de la lecture, Paris, Clancier-Guénaud, 1982, p. 32.

5. Voir Raymond Lebègue, "Réalisme et apprêt dans la langue des personnages de l'Heptaméron," dans La littérature narrative d'imagination, Paris, P.U.F., 1961, p. 73-86; cité par Cazauran, op. cit., p. 95.

6. Voir Pierre Jourda, Conteurs français du XVle siècle, préface, op.cit.

7. Gérard Genette, Figures III, Paris, Seuil, 1972, p. 261ss. Exemples de régie par Parlamente: "Et quant iil [Amadour] fut arrivé à Sauce, commencea la guerre grande et cruelle entre les deux Roys, laquelle ne suis deliberé de racompter, ne aussy les beaulx faictz que feit Amadour, car mon compte seroit assez long pour employer toute une journée." (p. 765).

8. Voir Deborah N. Losse, "Modes du récit dans la nouvelle française du seizième siècle," dans La Nouvelle, Montréal, Plato Academic Press, 1982, p. 208-215.

9. Marie-Madeleine de la Garanderie, Le dialogue des romanciers, une nouvelle lecture de l'Heptaméron, Paris, Archives des Lettres modernes, Minard, 1977, nº 168, p. 75

10. Terme utilisé par Philippe de Lajarte, qui désigne ainsi "tout discours exprimant une doxa, une opinion (vraie ou fausse)": voir "Modes du discours et formes d'altérité dans les 'Nouvelles' de Marguerite de Navarre," dans Littérature, $\mathrm{n}^{\circ}$ 55, octobre 1984, p. 71

11. "La fonction d'exemple, certes, n'est pas la seule qui soit dévolue aux nouvelles de l'Heptaméron. Celles-ci assument également, on l'a vu, une fonction esthético-affective dont on aurait tort de minimiser l'importance. Mais c'est leur fonction exemplaire qui, seule, établit un lien entre elles et les dialogues; c'est elle par conséquent qui fait du recueil de Marguerite de Navarre une oeuvre structurée." Ibid.

12. En fait, nous avons affaire à un discours paradoxal, à la fois ouvert et fermé, comme le montre bien Philippe de Lajarte, lequel, incidemment, conclut son article en établissant une relations entre l'Heptaméron et les Essais de Montaigne. Ibid., p. 72-73.

13. Montaigne, Oeuvres complètes, textes établis par A. Thibaudet et M. Rat, Paris, Gallimard, "Bibliothèque de la Pléiade," 1962. Mes références renvoient à cette édition.

14. Roland Barthes, "Introduction à l'analyse structurale des récits," dans Communications, n'8, Seuil, 1966, p. 4, n. 1 
15. Antoine Compagnon, "La brièveté de Montaigne," dans Les Formes brèves de la prose et le discours discontinu (XVIe-XVII siècles), p. 22.

16. Voir Ibid., p. 10

17. Voir Maynor Hardee, "Sur l'art narratif dans les anecdotes de Montaigne, dans le Bulletin de la Société des Amis de Montaigne, n 7-8, juillet-décembre 1981.

18. Hugo Friedrich, Montaigne, traduit de l'allemand par R. Rovini, Paris, Gallimard, "Bibliothèque des idées," 1968, p. 181. Voir aussi p. 168.

19. “Car les histoires que j'emprunte, je les renvoye sur la conscience de ceux de qui je les prens [...] Aussi en l'estude que je traitte de noz moeurs et mouvemens, les tesmoignages fabuleux, pourveu qu'ils soient possibles, y servent comme les vrais." I, 21, p. 104.

20. Voir Cazauran, op. cit., p. 33, n. 32.

21. Henri Coulet, Le roman jusqu'à la Révolution, Paris, Armand Colin, 1967. p. 128.

22. Expression utilisée par Simone de Reyff dans son introduction à l'Heptaméron. Paris, Flammarion, collection Garnier-Flammarion, 1982, p. 23

23. Voir Cazauran, op. cit., p. 82 et 95.

24. Rhétorique de l'essai littéraire, Montréal, Presses de l'Université du Québec, 1977, p. 22.

25. Jean Lafond, "Des formes brèves de la littérature morale aux XVIe et XVII ${ }^{\mathrm{e}}$ siècles," dans Les Formes brèves de la prose et le discours discontinu (XVI ${ }^{e}-X V I I^{e}$ siècles), Paris, Librairie philosophique J. Vrin, “De Pétrarque à Descartes,” 1984, p. 116.

26. Jean Lafond, “Avant-propos," op. cit., p. 7

27. Friedrich, op. cit., p. 30.

28. Marcel Tetel, op. cit., p. 3 et 106. Voir aussi From Marot to Montaigne, Lexington, French Forum, 1972, p. 125-135. 